\title{
Investigación sobre desplazamiento forzado en la localidad de Suba, Bogotá, 2012
}

\author{
Guillermo Restrepo Chavarriaga² \\ Alonso Belalcázar Urrea³ \\ Martha Isabel Sarmiento Osorio 4
}

doi:10.11144/Javeriana.ie17-2.idfl

Cómo citar: Restrepo Chavarriaga G, Belalcázar Urrea A, Sarmiento Osorio MI. Investigación sobre desplazamiento forzado en la localidad de Suba, Bogotá, 2012. Investig Enferm. Imagen Desarr. 2015;17(2): 145-155. http://dx.doi.org/10.11144/Javeriana.ie17-2.idfl

1. Artículo original de investigación. Recibido: 26 de noviembre de 2014. Aceptado: 11 de diciembre de 2014. Disponible en línea: 2 de mayo de 2015.

2. Médico cirujano. Magíster en Salud Pública. Profesor emérito. Director del Departamento de Planeación, Fundación Universitaria Juan N. Corpas, Bogotá, Colombia. Correo electrónico: guillermo.restrepo@juanncorpas.edu.co

3. Médico cirujano. Magíster en Salud Pública. Profesor titular, Departamento de Planeación, Fundación Universitaria Juan N. Corpas, Bogotá, Colombia. Correo electrónico: alonso. belalcazar@juanncorpas.edu.co

4. Comunicadora social y periodista. Coordinadora de Planeación, Fundación Universitaria Juan N. Corpas, Bogotá, Colombia. Correo electrónico: martha.sarmiento@juanncorpas. edu.co 


\section{Resumen}

Introducción: estudiar la problemática de las personas en condición de desplazamiento se constituye hoy en día en prioridad nacional, ya que es de máxima actualidad. Objetivo: obtener información en la localidad de Suba, Bogotá, sobre población en estado de desplazamiento, conformada por un conjunto de personas de diferentes edades y sexo, que representan diferentes grupos étnicos del país y diversas razas. Metodología: la selección de la muestra se realizó a través de un muestreo no probabilístico por conveniencia. La muestra fue constituida por 32 familias convocadas por la Fundación Salud, Familia y Comunidad. Las personas en condición de desplazamiento fueron encuestadas con el propósito de conocer condiciones previas y actuales. Resultados: las familias escogidas para este estudio estaban apoyadas por la La Unidad de Atención y Orientación a la Población desplazada, UAO Suba. Su principal característica, era la de ser personas en condición de desplazamiento forzado, provenientes siempre de la provincia colombiana. Conclusiones: importante proporción de las personas en condición de desplazamiento de este estudio, son provenientes del Pacifico Colombiano, siendo la principal causa de desplazamiento, el conflicto armado; En esta investigación encontramos que, la mayor parte del grupo desea establecerse en la capital, debido a las circunstancias de violencia vividas previas al desplazamiento. Las condiciones de servicios públicos ya encontrados en la ciudad, atraen su permanencia, el grupo que desea permanecer, aspira a mejorar sus condiciones de hacinamiento, y tener una mejor calidad de vida.

Palabras clave: conflicto armado; desplazamiento; familia; grupos armados; persona en situación de desplazamiento

\section{Research on forced displacement in Suba, Bogotá, 2012}

\section{Abstract}

Introduction: studying the problems of people living in displacement is today a national priority due to the fact that it is topical. Objective: To obtain information in the locality of Suba, Bogotá, about displaced population, which is comprised by people of different ages and gender, representing different ethnic groups and different races. Methodology: The sample selection was made through a non-probability sampling. The sample consisted of 32 families convened by the Foundation Salud, Familia y Comunidad. People in displacement conditions were surveyed in order to know previous and current conditions. Results: The families chosen for this study were supported by the Unit of Attention and Orientation to the displaced population, UAO Suba. Its main feature was to be people living in forced displacement from the province colombiana. Conclusions: always significant proportion of people living in displacement of this study are from the Colombian Pacific, the main cause of displacement, armed conflict; In this study we found that most of the group wants to settle in the capital, due to the circumstances of violence experienced prior to displacement. Utility conditions found in the city and attract their stay, the group that wants to stay, aspiring to improve their overcrowded, and have a better quality of life.

Keywords: armed conflict; displacement; family; armed groups; displaced person 


\section{Pesquisa sobre deslocamento forçado na localidade de Suba, Bogotá, 2012}

\section{Resumo}

Introdução: estudar a problemática do pessoal em condição de deslocamento constitui hoje uma prioridade nacional, pois ela é de máxima atualidade. Objetivo: obter informação na localidade de Suba, Bogotá, sobre população em estado de deslocamento, conformada por um conjunto de pessoas de diferentes idades e gênero, que representam diferentes grupos étnicos do país e diversas raças. Metodologia: a seleção da amostra foi realizada através de amostragem não probabilística por conveniência. A amostra foi constituída por 32 famílias convocadas pela Fundación Salud, Familia y Comunidad (Fundação Saúde, Família e Comunidade). As pessoas em condição de deslocamento responderam inquérito com o propósito de conhecer condições prévias e atuais. Resultados: As famílias escolhidas para este estudo foram apoiados pela Unidade de Atenção e Orientação à população deslocada, UAO Suba. Sua principal característica era ser pessoas que vivem em deslocamento forçado das colombianas. Conclusiones: província proporção sempre significativo de pessoas que vivem em deslocamento deste estudo são do Pacífico colombiano, a principal causa do deslocamento, conflito armado; Neste estudo verificou-se que a maior parte do grupo quer resolver na capital, devido às circunstâncias de violência vividos antes da deslocação. Condições de utilidade encontrado na cidade e atrair a sua estadia, o grupo que quer ficar, aspirando a melhorar a sua superlotadas, e ter uma melhor qualidade de vida.

Palavras-chave: conflito armado; deslocamento; família; grupos armados; pessoa em situação de deslocamento 


\section{Introducción}

Desde hace más de medio siglo, nuestro país ha venido sufriendo de un fenómeno de violencia, el cual ha provocado un desplazamiento de personas, familias, grupos y aun comunidades, "a áreas suburbanas, que incrementan la aglomeración e invasiones; con una población que en búsqueda de mejores condiciones de trabajo, educación y de salud; han transformado la población rural, a un altísimo porcentaje de habitantes urbanos, viviendo en muy malas condiciones, según el reporte de la Encuesta Demográfica Nacional del 2011" (1).

Las familias en condición de desplazamiento, procedentes de la provincia colombiana, presentan en su gran mayoría características similares en cuanto a condiciones de modus vivendi y han buscado básicamente una solución a su problemática en organizaciones gubernamentales y no gubernamentales.

La Unidad de Atención y Orientación a la Población desplazada (UAO) les brinda apoyo para cumplir su misión. Según el informe oficial de la Alcaldia Mayor de Bogotá, con ayuda humanitaria de urgencia, documentos de identidad, salud, educación, vivienda, protección de tierras y patrimonio, asesoría jurídica seguridad y protección, a cargo de la Secretaría Distrital de Integración Social y Generación de Ingresos, Familias en Acción.

Este estudio permite conocer el pensamiento futuro de las personas en condición de desplazamiento, lo que es de utilidad para las organizaciones que buscan proporcionar apoyo. En particular, esta observación y las condiciones de vivienda orientan a los miembros de la UAO y de la Corporación Cultural Nueva Tibabuyes (Cultiba), para las actividades que ellos realizan.

Dentro de los conceptos básicos de la política pública de retornos, según el artículo 1 de la Ley 387 de 1997, una persona en situación de desplazamiento es aquella que:

[...] se ha visto forzada a migrar dentro del territorio nacional, a abandonar su localidad de residencia y las actividades económicas habituales porque su vida, su integridad física, su seguridad o libertad personal han sido vulneradas o se encuentran directamente amenazadas. Las razones que conducen al desplazamiento son: conflicto armado interno, disturbios y tensiones interiores, violencia generalizada, violaciones masivas de los derechos humanos, infracciones al Derecho Internacional Humanitario u otras circunstancias emanadas de las situaciones anteriores que puedan alterar o alteren drásticamente el orden público. (2)

Acorde con la propuesta del Departamento Administrativo de Ciencia y Tecnología e Innovación (3), debe ser la investigación un trabajo conjunto entre entidades gubernamentales y universidades, para suministrar al país una visión integral del fenómeno y aportar no solamente mediciones del problema actual, sino posibles politicas, estrategias y tácticas por seguir, para manejarlo y solucionarlo. Dado lo anterior se investigó, en la localidad mencionada, la procedencia de las personas en condición de desplazamiento y su causa. 


\section{Metodologia}

El diseño utilizado fue descriptivo. El tamaño de la muestra correspondió a 32 personas de diferentes razas, provenientes de diferentes lugares del país, obtenido a través de un muestreo no probabilístico por conveniencia. Los criterios de inclusión fueron: residentes de Suba, que asistían a la Fundación Salud Familia y Comunidad, en condición de desplazamiento.

Para la recolección de la información se diseñó una encuesta que contenía la siguiente información: lugar de residencia de las familias antes de generarse el desplazamiento, principal causa que originó el desplazamiento, conocer si contaban con alguna red de apoyo durante el desplazamiento, conocer si desean regresar a su ciudad de origen o establecerse en la ciudad, disponibilidad de servicios públicos de las familias antes del desplazamiento y después de este.

Posterior a esto, se realizó una prueba piloto, la cual se aplicó a cinco personas sin necesidad de modificar las preguntas de las encuestas. Se facultó a un grupo de ocho de encuestadores que fueron previamente capacitados y se les explicó los sinónimos regionales.

Para la aplicación de la encuesta se informó previamente sobre los componentes de esta y se solicitó la firma del consentimiento informado. Para la elaboración de la prueba piloto se conversó con la coordinadora de la UAO y se le explicó el trabajo que se iba a realizar, y así obtener el acercamiento a las familias, realizarla y conseguir la comprobación y correcta comprensión de las preguntas, sin necesidad de modificar las encuestas, llevadas a cabo durante dos sesiones.

Los encuestadores fueron instruidos y capacitados para exponer bien la encuesta y su objetivo a cada una de las familias. Se aplicó primordialmente a personas cabeza de familia; luego se organizaron los resultados de la información obtenida en las encuestas, con el fin de emplearlos como respaldo y conocer las condiciones de los migrantes.

Se utilizó un programa estadístico de Excel®, y con los resultados se elaboraron las tablas que permiten ver los porcentajes que muestran las tendencias que afectan a las personas en condición de desplazamiento.

\section{Resultados}

Se analizaron las encuestas aplicadas a las 32 familias y se obtuvo la siguiente información:

En la tabla 1, destaca el Departamento de Chocó como la principal fuente de desplazamiento en el ámbito nacional (21,88\%). Otras áreas del Pacífico estuvieron presentes. El Tolima tuvo amplia representación con un 12,50\%.

TABLA 1. Lugar de residencia de las familias antes de generarse el desplazamiento

\begin{tabular}{|c|c|c|}
\hline Lugar & Número personas & Porcentaje \\
\hline Chocó & 7 & 21,8 \\
\hline Tolima & 4 & 12,5 \\
\hline
\end{tabular}




\begin{tabular}{|c|c|c|}
\hline Lugar & Número personas & Porcentaje \\
\hline Bolívar & 3 & 9,3 \\
\hline Córdoba & 3 & 6,3 \\
\hline Cauca & 2 & 6,2 \\
\hline Huila & 2 & 6,2 \\
\hline Cesar & 2 & 6,2 \\
\hline Meta & 1 & 3,1 \\
\hline Cundinamarca & 1 & 3,1 \\
\hline Antioquia & 1 & 3,1 \\
\hline Barrio en Bogotá & 1 & 3,1 \\
\hline Cúcuta & 1 & 3,1 \\
\hline Buenaventura & 1 & 100 \\
\hline Magdalena & 32 & \\
\hline Total & 1 & 3,1 \\
\hline
\end{tabular}

Fuente: elaboración propia con base en los datos del estudio

Es importante destacar, como se observa en la tabla 2, que la principal causa que originó el desplazamiento fueron los grupos armados, con 28 (del total de integrantes de las 32 familias).

TABLA 2. Principal causa que originó el desplazamiento de las familias $(n=32)$

\begin{tabular}{|l|c|}
\hline \multicolumn{1}{|c|}{ Causa } & Número \\
\hline Grupo armado & 28 \\
\hline Educación & 6 \\
\hline Desempleo & 5 \\
\hline Salud & 2 \\
\hline Incendio & 1 \\
\hline Problemas & 1 \\
\hline Total & 43 \\
\hline
\end{tabular}

Fuente: elaboración propia con base en datos del estudio 
Como se presenta en la tabla 3, este grupo de personas en condición de desplazamiento encontró apoyo primordialmente en redes de apoyo oficiales y privadas.

TABla 3. Redes de apoyo de las familias en condición de desplazamiento, localidad de Suba, 2012

\begin{tabular}{|l|c|}
\hline \multicolumn{1}{|c|}{ Red de apoyo } & Si cuenta con esta \\
\hline Oficiales & 12 \\
\hline Privadas & 9 \\
\hline No reportaron & 6 \\
\hline Familiares & 3 \\
\hline Otros (ONG o fundaciones) & 2 \\
\hline
\end{tabular}

Fuente: elaboración propia con base en datos del estudio

La meta del $68,75 \%$ de las personas en situación de desplazamiento del grupo encuestado es establecerse en la ciudad de Bogotá, y la del 31,25\% es regresar al sitio de origen (tabla 4).

TABLA 4. Consideraciones a futuro de las personas en situación de desplazamiento

\begin{tabular}{|l|c|}
\hline \multicolumn{1}{|c|}{ Consideración a futuro } & Número de personas \\
\hline Establecerse en la ciudad & 22 \\
\hline Regresar al sitio de origen & 10 \\
\hline
\end{tabular}

Fuente: elaboración propia con base en datos del estudio

En la tabla 5 se presenta la utilización de los servicios públicos con los que cuenta actualmente cada familia en situación de desplazamiento.

Tabla 5. Disponibilidad de servicios públicos de las familias en situación de desplazamiento

\begin{tabular}{|l|c|c|c|c|}
\hline \multirow{2}{*}{ Servicios públicos } & \multicolumn{2}{c|}{ Anteriormente } & \multicolumn{2}{c|}{ Actualmente } \\
\cline { 2 - 5 } & Si & No & Si & No \\
\hline Energía eléctrica & 26 & 6 & 32 & \\
\hline Agua & 21 & 11 & 32 & \\
\hline Alcantarillado & 11 & 21 & 32 & \\
\hline Recolección de basura & 10 & 22 & 32 & \\
\hline Gas & 5 & 27 & 30 & 2 \\
\hline Teléfono & 3 & 29 & 6 & 26 \\
\hline
\end{tabular}

FUente: elaboración propia con base en datos del estudio 
Los encuestadores observaron que en una sola habitación dormían cuatro o más personas, en un espacio aproximado de seis metros cuadrados, en más de la mitad de las familias visitadas.

\section{Discusión}

Los resultados coinciden con otras publicaciones, e informes frecuentes de prensa, que señalan que gran proporción de nuestros desplazados vienen a las ciudades desde nuestro Pacífico colombiano (4). Se observó que alrededor de dos terceras partes tuvieron como razón principal para refugiarse en la ciudad el conflicto armado, que los amenaza permanentemente y los obliga a cambiar su estilo de vida. Otras razones, en menores proporciones, fueron educación y desempleo.

"La tasa de deserción en el Chocó es del 18 por ciento en primaria y del 27 por ciento en secundaria. El 80 por ciento de los niños del Chocó respondió que habían desertado por falta de alimentación escolar" (5). Lo anterior coincide con el periódico de la región, en los aspectos educacionales de ese departamento.

Según el Departamento Administrativo Nacional de Estadística (DANE), en su informe de prensa, "Principales indicadores del mercado laboral departamentos-2012", señala que "La tasa de subempleo subjetivo de Chocó fue 23,5\%, superior en 1,0 puntos porcentuales frente al año anterior $(22,5 \%)$ y en Quibdó la tasa de desempleo era del 21,5\%" (6).

En cuanto a vivienda, las dos terceras partes de ellos no quieren regresar a sus terruños y "se meten en un cuarto". Los que quepan, de cualquier forma, se acondicionan, con las consecuencias del hacinamiento (7).

Al comparar estos resultados con un estudio de caso realizado en localidades de Suba y Ciudad Bolivar, publicado por la Oficina en Colombia del Alto Comisionado de las Naciones Unidas para los Refugiados (8), se llega a una misma conclusión que el estudio referido sobre Suba, donde "la mayoría de personas en condición de desplazamiento provienen del Pacífico colombiano, zona de alto índice de pobreza, y casos de enfermedades transmisibles" (9) y de violencia marcada por presencia de grupos armados. Así, estas se postulan como causa de desplazamiento.

En cuanto a salud, las personas en condición de desplazamiento no han logrado resolver sus relaciones con la entidad promotora de salud (10), es decir, no cuentan con servicios médicos de forma integral; afortunadamente, la Fundación Salud Familia y Comunidad, con el apoyo de la Fundación Universitaria Juan N. Corpas, suministra adecuadamente salud a sus familias; pero no plenamente, ya que no puede ubicarlos con oportunidad, en niveles superiores de atención, cuando la salud lo requiere.

La definición más ambiciosa de la salud fue propuesta por la Organización Mundial de la Salud, en 1946, que la define "Como un estado de completo bienestar físico, mental y social y no meramente la ausencia de enfermedad o dolencia" (11). Estas personas en situación de desplazamiento necesitan definiciones de salud y enfermedad más prácticas. Una de ellas es como la planteada por Leavell y Clark (12), que nos recuerda la historia natural de la enfermedad y la cadena epidemiológica: agente causal, reservorio, 
modo de trasmisión del agente, puerta de eliminación o salida del agente, puerta de entrada en el huésped y huésped susceptible. Nuestras personas en situación de desplazamiento están expuestas a que esta cadena se cumpla y se enfermen en un medio que no es el de ellos.

También se debe tener en cuenta que la intención de la Ley 1438 de $2011(13,14)$ y la Ley Estatutaria en Salud del 2013 (14) es lograr total cobertura, y sirven como máximo apoyo para la estrategia de atención primaria, que cobija a todas las personas en situación de desplazamiento.

Se corrobora que estas familias colombianas están viviendo en condiciones más inestables que cuando vivían en sus casas originarias, de las cuales fueron desplazadas y, basándonos en esto, concordamos con Carlos Alberto Tovar y Johanna Eloísa Vargas, "en el sentido, que esta población es víctima de una guerra que se refugia en la ciudad, con grandes objetivos de mejorar su vida, educación y salud sin encontrar mayor ayuda" (15).

Según la información obtenida de las encuestas de Suba, 19 de 32 familias están de acuerdo con que la atención primaria en salud brindada es oportuna. De estas 32 familias, 7 no están afiliadas a ninguna entidad promotora de salud, lo que lleva a pensar en los diferentes problemas de salud física y mental ocultos en una magnitud mayor de la población en situación de desplazamiento, y que una intervención multisectorial no será suficiente mientras no haya reincorporación social de las personas en situación de desplazamiento.

Otras necesidades, como empleo, alimentación, seguridad, restitución de tierras y oportunidades, deben darse, pero por tiempos determinados, para evitar que se queden permanentemente en esta condición y, más temprano que tarde, se constituya en fuerza de desarrollo para el país. Hasta ahora muchos de los migrantes no hacen mayores esfuerzos por regresar a la vida laboral.

Esta indefinición por no tomar nuevos rumbos hace que permanezcan indefinidamente en los programas del gobierno, y con ello le quitan la oportunidad de acceder a estos a nuevas personas que lo requieran.

\section{Conclusiones}

Se estudió un grupo de personas en condición de desplazamiento en la localidad de Suba provenientes primordialmente del Departamento del Chocó, por causa del conflicto armado. En las personas en condición de desplazamiento, a pesar del transcurrir del tiempo, no se observa ánimo de definir su futuro y su ubicación. Dentro del grupo las dos terceras partes manifestó el ánimo de quedarse, y una tercera parte, el deseo de regresar.

\section{Recomendación}

El grupo de investigación sobre Modelos de Atención de la Fundación Universitaria Juan N. Corpas recomienda considerar que estos datos obtenidos se deben tener en cuenta para las definiciones de políticas, para las personas en situación de desplazamiento, y deben unirse a otros que adelanten o hayan adelantado en el Gobierno Nacional u otras universidades, para el adecuado manejo del desplazamiento (16). 


\section{Agradecimientos}

A las instituciones que nos prestaron su colaboración: a la Unidad de Atención al desplazado (UAO, oficina oficial del Distrito); a la Oficina en Colombia del Alto Comisionado de las Naciones Unidas para los Refugiados (Acnur); a la Corporación Cultural Nueva Tibabuyes (Cultiba), y a los estudiantes de la Facultad de Medicina Juan N. Corpas en el segundo semestre del 2011, semilleros de investigación: Mairlys Shakira Álvarez Ortiz, Lina Silvana Arce Celis y Alfonso Leonardo Camacho Góngora.

\section{Conflicto de interés}

Ninguno

\section{Financiación}

Ninguno

\section{Referencias}

1. Situación Colombia panorama regional [internet]. 2012 [citado 14 sep. 2012]; [aprox.4p]. Disponible en: http://www.acnur.org/t3/fileadmin/Documentos/RefugiadosAmericas/Colombia/2012/Situacion_ Colombia_-_Agosto_2012.pdf?view=1

2. Ley 387 de 1997/de julio 18, por medio de la cual se adoptan medidas para la prevención del desplazamiento forzado; la atención, protección, consolidación y estabilización socioeconómica de los desplazados internos por la violencia en la República de Colombia (título1, artículo 1) [internet]. Disponible en: http://www.acnur.org/t3/filead$\mathrm{min} / \mathrm{scripts} /$ doc.php?file=biblioteca $/ \mathrm{pdf} / 1295$

3. Colciencias. Ciencia, tecnología e innovación en educación [internet]. 2012 [citado junio 2012]; [aprox. 2 p.]. Disponible en: http:// www.colciencias.gov.co/programa_estrategia/programanacional-de-estudios-cient-ficos-de-la-educaci-n-0

4. Asociación Pro Bienestar de la Familia Colombiana (Profamilia). Encuesta en zonas marginales salud sexual y salud reproductiva, desplazamiento forzado y pobreza. 2000-2011. Disponible: http:// www.profamilia.org.co/encuestaenzonasmarginadas/pdf/ezm2011_ contexto.pdf

5. Rueda Castañeda R. Chocó: la educación. Diario Chocó 7 Días [internet]. 2012, 7 de mayo [citado julio 2012]. Disponible en: http://rennyrueda.com/2012/09/17/choco-la-educacion/

6. Departamento Administrativo Nacional de Estadística (DANE). Principales indicadores del mercado laboral departamentos [internet]. 2012. Disponible en: http://www.dane.gov.co/files/investigaciones/ boletines/ech/ml_depto/Boletin_dep_12.pdf

7. Organización Panamericana de la Salud (OPS). Módulo de principios de epidemiología para el control de enfermedades. $2^{\text {a }}$ ed. Washington: OPS; 2002.

8. Vidal López R, Atheortúa Arredondo C, Salcedo J. Efectos del desplazamiento interno en las comunidades de las zonas de recepción: 
estudio de caso en Bogotá, DC Colombia, en las localidades de Suba y Ciudad Bolivar. s. d.; 2011.

9. Coordination Saves Lives, OCHA. Desplazamiento y emergencia en salud: medio San Juan [internet]. 2012 [citado 14 sep 2012]; [aprox. 3 p]. Disponible en: http://www.colombiassh.org/site/IMG/ pdf/120423_Desplazamiento_Medio_San_Juan_revisado_MJ.pdf

10. Ley 100 de 1993, título II: la organización del Sistema General de Seguridad Integral.

11. Organización Mundial de la Salud (OMS). Definición de salud, 1946. s. d.

12. Leavell H, Clark E. Preventive medicine for doctor in his community and epidemiologic approach: The doctor in community. New York: McGraw Hill; 1965. pp. 1-684.

13. Ley 1438 de 2011 /de 19 de enero, por medio de la cual se reforma el Sistema General de Seguridad Social en Salud y se dictan otras disposiciones. Capítulo II, título II (La organización del Sistema General de Seguridad Integral). Diario Oficial 47.957 (19-01-2011).

14. Ley 1438 de $2011 /$ de 19 de enero, por medio de la cual se reforma el Sistema General de Seguridad Social en Salud y se dictan otras disposiciones. Capítulo III (atención primaria en salud). Diario Oficial 47.957 (19-01-2011).

15. Torres CA, Vargas JE. Vivienda para población desplazada en Colombia: recomendaciones para la politica pública y exigibilidad del derecho. Rev. INVI. 2009;24(66):17-86.

16. Ley Estatutaria 2009 de 2013, por medio de la cual se regula el derecho fundamental a la salud y se dictan otras disposiciones. 
